\title{
Inclusion of lactate level measured upon emergency room arrival in trauma outcome prediction models improves mortality prediction: a retrospective, single-center study
}

\author{
Jonghwan Moon', Kyungjin Hwang', Dukyong Yoon², Kyoungwon Jung ${ }^{1}$ \\ ${ }^{1}$ Division of Trauma Surgery, Department of Surgery, Ajou University School of Medicine and Graduate School of Medicine, Suwon; ${ }^{2}$ Department of Biomedical \\ Informatics, Ajou University School of Medicine and Department of Biomedical Sciences, Ajou University Graduate School of Medicine, Suwon, Korea
}

Background: This study aimed to develop a model for predicting trauma outcomes by adding arterial lactate levels measured upon emergency room (ER) arrival to existing trauma injury severity scoring systems.

Methods: We examined blunt trauma cases that were admitted to our hospital during 20102014. Eligibility criteria were cases with an Injury Severity Score of $\geq 9$, complete Trauma and Injury Severity Score (TRISS) variable data, and lactate levels that were assessed upon ER arrival. Survivor and non-survivor groups were compared and lactate-based prediction models were generated using logistic regression. We compared the predictive performances of traditional prediction models (Revised Trauma Score [RTS] and TRISS) and lactate-based models using the area under the curve (AUC) of receiver operating characteristic curves.

Results: We included 829 patients, and the in-hospital mortality rate among these patients was $21.6 \%$. The model that used lactate levels and age provided a significantly better AUC value than the RTS model. The model with lactate added to the TRISS variables provided the highest Youden J statistic, with $86.0 \%$ sensitivity and $70.8 \%$ specificity at a cutoff value of 0.15 , as well as the highest predictive value, with a significantly higher AUC than the TRISS.

Conclusions: These findings indicate that lactate testing upon ER arrival may help supplement or replace traditional physiological parameters to predict mortality outcomes among Korean trauma patients. Adding lactate levels also appears to improve the predictive abilities of existing trauma outcome prediction models.

Key Words: lactate; mortality; prognosis; wounds and injuries

\section{INTRODUCTION}

It is often difficult to determine the severity of multiple blunt trauma based on a patient's appearance, and under-triage of trauma during the initial resuscitation process can lead to death. Thus, models have been developed to identify the severity of injuries [1-10], including trauma outcome prediction models that use statistical methods (i.e., logistic regression), to quantify diverse and complex traumas and estimate the likelihood of mortality. These models facilitate better communication among medical staff and improve treatment prioritization.

\section{Original Article}

Received: December 9, 2019

Revised: May 3, 2020

Accepted: May 19, 2020

Corresponding author

Kyoungwon Jung

Division of Trauma Surgery,

Department of Surgery, Ajou

University School of Medicine and

Graduate School of Medicine, 206

World cup-ro, Yeongtong-gu, Suwon

16499, Korea

Tel: +82-31-219-7764

Fax: +82-31-219-7781

E-mail: jake98@daum.net

Copyright $\odot 2020$ The Korean Society of Critical Care Medicine

This is an Open Access article distributed under the terms of Creative Attributions Non-Commercial License (https:// creativecommons.org/li-censes/by-nc/4.0/) which permits unrestricted noncommercial use, distribution, and reproduction in any medium, provided the original work is properly cited. 
The Revised Trauma Score (RTS) combines clinical and observational physiologic data into a single score. The RTS incorporates the Glasgow Coma Scale (GCS), systolic blood pressure (SBP), and respiratory rate (RR). The RTS is strongly correlated with mortality and remains important in injury scoring through its contribution to the Trauma and Injury Severity Score (TRISS) model. The TRISS is a weighted combination of patient age, Injury Severity Score (ISS), and RTS variables that predicts a patient's probability of survival (Ps). Using the TRISS methodology, the Ps of any one patient can be estimated using the following formula:

$$
\text { Ps }=1 /\left(1+\mathrm{e}^{-\mathrm{b}}\right)
$$$$
\text { where } b=b_{0}+b_{1} \text { (RTS) }+b_{2} \text { (ISS) }+b_{3} \text { (age). }
$$

Here, $b_{0-3}$ are coefficients derived from Walker-Duncan regression analysis applied to data from thousands of patients analyzed in the Major Trauma Outcome Study [10].

The TRISS considers the trauma's anatomical location, as well as the patient's age and physiological parameters, which makes it a useful system for predicting trauma outcomes [1012]. However, the TRISS requires both accurate Abbreviated Injury Scale (AIS) coding and reliable information regarding initial SBP, RR, and GCS to calculate the RTS. Furthermore, previous studies have revealed that data are missing for approximately $20 \%$ of the variables, which highlights the difficulty in obtaining reliable information regarding these values $[11,13,14]$. This problem is magnified in Korea, where the trauma management system is relatively new, and patients are often transferred to a hospital without appropriate triage during initial resuscitation. Therefore, it is extremely difficult to obtain accurate physiological values, and AIS coding is only available at certain hospitals with trained staff. Moreover, the lack

\section{KEY MESSAGES}

- Our findings suggest that lactate testing at emergency room arrival may help supplement or replace traditional physiological parameters for prognostication in Korean trauma cases.

of post-mortem examination, which is related to cultural preferences, also interferes with accurate AIS coding. Thus, it has been difficult to use established tools to predict outcomes in Korean trauma cases.

Recent studies have indicated that lactate can be a useful marker for predicting trauma outcomes. We hypothesized that lactate levels, which reflect organ perfusion, could help determine the degree of anatomical trauma and/or the patient's physiological parameters using a single examination. Therefore, our goal was to develop a model for predicting trauma outcomes by assessing the relationship between arterial lactate level upon emergency room (ER) arrival and outcomes in cases of multiple blunt trauma.

\section{MATERIALS AND METHODS}

\section{Research Ethics}

This study's retrospective protocol was approved by the Institutional Review Board of Ajou University Hospital (IRB No. AJIRB-MED-MDB-17-359). Informed consent was waived by the board because of the observational nature of the study.

\section{Setting and Patient Data}

We examined data from trauma cases that were treated at our

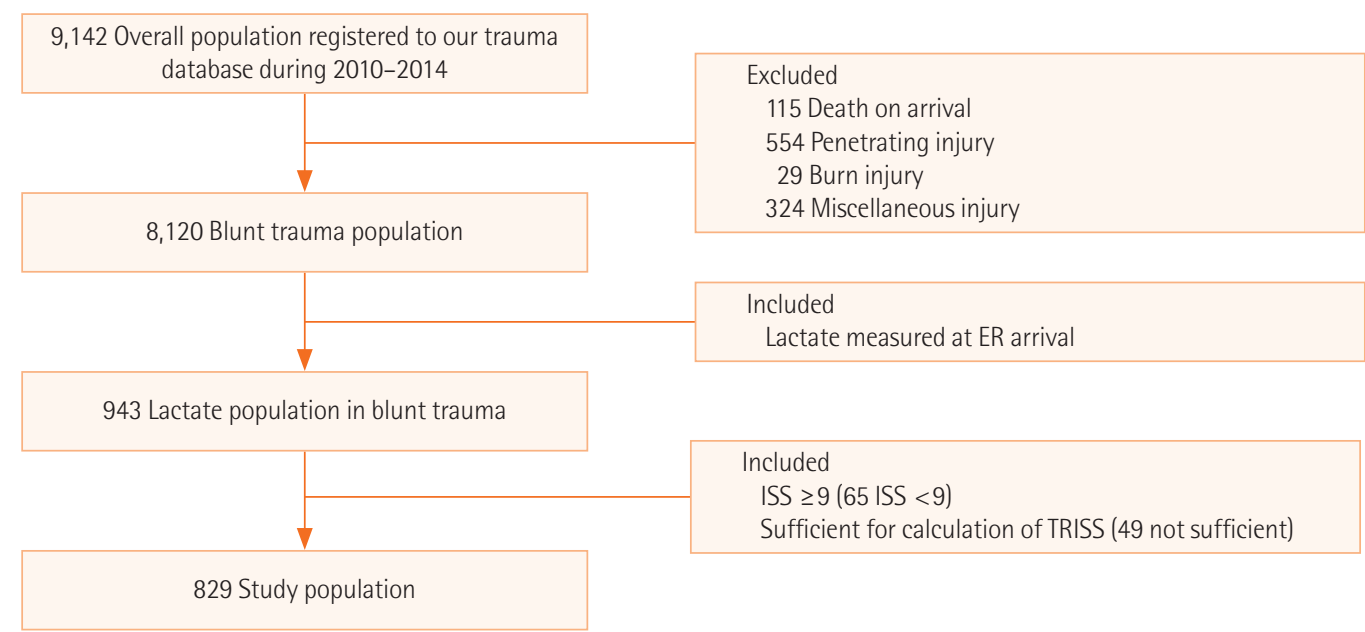

Figure 1. Study population. ER: emergency room; ISS: Injury Severity Score; TRISS: Trauma and Injury Severity Score. 
center between 2010 and 2014. Among the in-patient trauma cases, we targeted cases where the mechanism of injury was blunt force. We excluded patients who were dead on arrival or missing lactate data at the time of ER arrival. As the main outcome of interest was in-hospital mortality, this study was designed to compare our new lactate-based scoring system and a representative trauma mortality prediction model, the TRISS. Therefore, only cases with an ISS of $\geq 9$ and complete TRISS variable data were considered eligible for inclusion (Figure 1).

\section{Variables for the Prediction Models}

Lactate levels were assessed using arterial blood upon ER ar- rival, and the interval between ER arrival and blood sampling was calculated. Patients were divided into four groups based on their lactate levels: $\leq 2.5,2.6-5.0,5.1-7.5$, and $\geq 7.6 \mathrm{mmol} / \mathrm{L}$. According to the TRISS method, we also dichotomized patients based on an age of 55 years. RTS was calculated based on SBP, RR, and the GCS at ER arrival, which were categorized based on the RTS variables' breakpoints. AIS coding was performed by a well-trained trauma nurse, and we carefully evaluated and confirmed the results.

\section{Statistical Analysis}

Survivor and non-survivor groups were compared using the

Table 1. Comparison of TRISS predictor variables and initial lactate values for the study population

\begin{tabular}{|c|c|c|c|c|}
\hline Variable & All patients $(\mathrm{N}=829)$ & Survivor $(n=650)$ & Non-survivor $(n=179)$ & P-value \\
\hline Age (yr) & $47(31-58)$ & $45(30-56)$ & $52(36-65)$ & $<0.001$ \\
\hline $0-55$ & $565(68.2)$ & $465(71.5)$ & $100(55.9)$ & $<0.001$ \\
\hline$>55$ & 264 (31.8) & $185(28.5)$ & $79(44.1)$ & \\
\hline ISS & $25(16-29)$ & $22(16-27)$ & $29(25-34.75)$ & $<0.001$ \\
\hline $\mathrm{SBP}$ at arrival $(\mathrm{mm} \mathrm{Hg})$ & $120(110-139)$ & $120(101-139)$ & $110(80-140)$ & 0.010 \\
\hline 0 & $7(0.8)$ & $1(0.2)$ & $6(3.4)$ & $<0.001$ \\
\hline $1-49$ & $3(0.4)$ & 0 & $3(1.7)$ & \\
\hline $50-75$ & $58(7.0)$ & $31(4.8)$ & $27(15.1)$ & \\
\hline $76-89$ & $67(8.1)$ & $49(7.5)$ & $18(10.1)$ & \\
\hline$>89$ & 694 (83.7) & $569(87.5)$ & 125 (69.8) & \\
\hline RR at arrival (/min) & $18(16-23)$ & $18(15-22)$ & $23(16-25)$ & 0.013 \\
\hline 0 & $7(0.8)$ & $1(0.2)$ & $6(3.4)$ & $<0.001$ \\
\hline $1-5$ & 0 & 0 & 0 & \\
\hline $6-9$ & $2(0.2)$ & $1(0.2)$ & $1(0.6)$ & \\
\hline$>29$ & $66(8.0)$ & $48(7.4)$ & $18(10.1)$ & \\
\hline $10-29$ & 754 (91.0) & $600(92.3)$ & 154 (86.0) & \\
\hline GCS at arrival & $15(7.75-15)$ & $15(11-15)$ & $6(3-12)$ & $<0.001$ \\
\hline 3 & $67(8.1)$ & $21(3.2)$ & $46(25.7)$ & $<0.001$ \\
\hline $4-5$ & $62(7.5)$ & $30(4.6)$ & $32(17.9)$ & \\
\hline $6-8$ & $101(12.2)$ & $67(10.3)$ & $34(19.0)$ & \\
\hline $9-12$ & 106 (12.8) & $83(12.8)$ & $23(12.8)$ & \\
\hline $13-15$ & $493(59.5)$ & $449(69.1)$ & $44(24.6)$ & \\
\hline RTS at arrival & $7.84(5.97-7.84)$ & $7.84(6.82-7.84)$ & $5.97(4.23-7.7)$ & $<0.001$ \\
\hline Initial lactate at ER (mmol/L) & $2.8(1.5-4.6)$ & $2.4(1.3-3.8)$ & $5.5(3.3-7.8)$ & $<0.001$ \\
\hline$\leq 2.5$ & $383(46.2)$ & $355(54.6)$ & $28(15.6)$ & $<0.001$ \\
\hline $2.6-5.0$ & $264(31.8)$ & $211(32.5)$ & $53(29.6)$ & \\
\hline $5.1-7.5$ & $120(14.5)$ & 70 (10.8) & $50(27.9)$ & \\
\hline$\geq 7.6$ & $62(7.5)$ & $14(2.2)$ & $48(26.8)$ & \\
\hline
\end{tabular}

Values are presented as median (interquartile range) or number (\%).

TRISS: Trauma and Injury Severity Score; ISS: Injury Severity Score; SBP: systolic blood pressure; RR: respiratory rate; GCS: Glasgow Coma Scale; RTS: Revised Trauma Score; ER: emergency room. 
Mann-Whitney U-test (non-normally distributed continuous variables), the chi-square test (categorical variables), or the chi-square test for trend (categorical variables with three or more groups). Lactate-based prediction models were generated using logistic regression analysis, with mortality as the dependent variable and lactate level, RTS, ISS, and age as the independent variables. Subsequently, we designed several new prediction models: the LA model, based on lactate-only, the LAG model based on lactate level and age, the LAGISS model that used lactate instead of the RTS (i.e., lactate and ISS), the LAGRTS model that used lactate and the RTS, and the LATRISS model that used lactate in addition to the traditional TRISS variables. The predictive value of each model was evaluated by calculating the area under the curve (AUC) of the receiver operating characteristic curve and the HosmerLemeshow test. Sensitivity, specificity, the Youden J value, and optimal cutoff value for outcome prediction were also obtained for each model. Predictive performances of the traditional prediction models (RTS and TRISS) and the lactate-based models were also compared. All analyses were performed using MedCalc software ver. 17.9.2 (MedCalc Software, Mariakerke, Belgium), and differences were considered statistically significant at $\mathrm{P}$-values of $<0.05$.

\section{RESULTS}

The present study included 829 patients ( 630 men) with a median age of 47 years. Approximately $55 \%$ of the patients ( 456 patients) were transferred to our center from a different hospital, and the in-hospital mortality rate was $21.6 \%$ (179/829). Significant differences in age, ISS, SBP, RR, GCS, RTS, and initial lactate levels were observed when we compared patients who survived with those who died. These differences remained significant after coding according to the RTS variable breakpoints (Table 1). Lactate levels were assessed at a median interval of 12 minutes (interquartile range, 8-21 minutes) after ER arrival. Surviving patients had a median lactate level of 2.4 $\mathrm{mmol} / \mathrm{L}$ while patients who died had a median lactate level of $5.5 \mathrm{mmol} / \mathrm{L}(\mathrm{P}<0.001)$. Higher lactate levels were positively correlated with mortality $(\mathrm{P}<0.001)$ (Figure 2$)$.

All age-based logistic regression models had AUC values of $\geq 0.8$, and the Hosmer-Lemeshow test results revealed that all models had P-values of $\geq 0.05$ (Table 2). The AUC of the traditional RTS was 0.735 , while that of the LA model was slightly higher, albeit not significantly so $(0.776, \mathrm{P}=0.151)$. The LAG model based on lactate levels and age had a significantly better AUC value than the traditional RTS $(0.803, \mathrm{P}=0.015)$ (Table

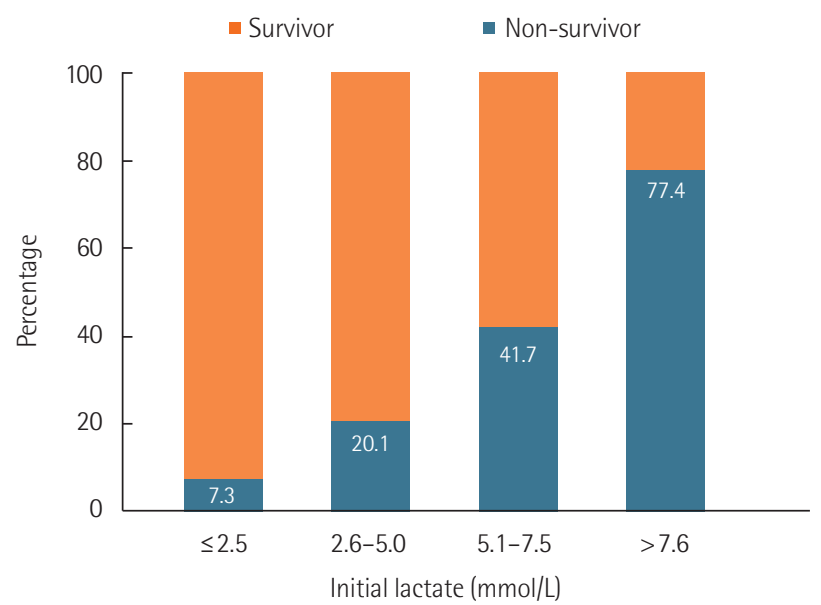

Figure 2. Trauma outcomes in relation to initial lactate levels. Patients that died and those that survived had significantly different lactate levels $(\mathrm{P}<0.001)$.

Table 2. Prediction model performance for mortality

\begin{tabular}{|c|c|c|c|c|c|c|}
\hline $\begin{array}{l}\text { Prediction model and variable } \\
\text { used in the models }\end{array}$ & $\begin{array}{l}\text { Discrimination AUC } \\
\qquad\left(95 \% \mathrm{Cl}^{\mathrm{a}}\right)\end{array}$ & $\begin{array}{l}\text { Calibration } \mathrm{H}-\mathrm{L} \\
\text { P-value }\end{array}$ & $\begin{array}{l}\text { Sensitivity } \\
(\%)\end{array}$ & $\begin{array}{l}\text { Specificity } \\
(\%)\end{array}$ & $\begin{array}{l}\text { Youden } \\
\text { Index J }\end{array}$ & $\begin{array}{l}\text { Cutoff } \\
\text { value }\end{array}$ \\
\hline RTS (SBP, RR, GCS) & $0.735(0.704-0.765)$ & 1.000 & 63.1 & 76.9 & 0.401 & 0.216 \\
\hline LA (lactate) & $0.776(0.746-0.804)$ & 1.000 & 54.8 & 87.1 & 0.418 & 0.199 \\
\hline LAG (lactate, age) & $0.803(0.774-0.829)$ & 1.000 & 67.6 & 80.0 & 0.476 & 0.149 \\
\hline TRISS (age, ISS, RTS) & $0.801(0.772-0.828)$ & 0.397 & 77.1 & 70.5 & 0.476 & 0.192 \\
\hline LAGISS (age, ISS, lactate) & $0.836(0.809-0.861)$ & 0.251 & 78.8 & 74.2 & 0.529 & 0.183 \\
\hline LAGRTS (age, RTS, lactate) & $0.849(0.823-0.873)$ & 0.183 & 83.2 & 71.9 & 0.551 & 0.170 \\
\hline LATRISS (age, ISS, RTS, lactate) & $0.859(0.833-0.882)$ & 0.603 & 86.0 & 70.8 & 0.568 & 0.150 \\
\hline
\end{tabular}

AUC: area under the receiver operator characteristic curve; Cl: confidence interval; H-L: Hosmer and Lemeshow test; RTS: Revised Trauma Score; SBP: systolic blood pressure; RR: respiratory rate; GCS: Glasgow Coma Scale; LA: lactate; LAG: lactate plus age; TRISS: Trauma and Injury Severity Score; ISS: Injury Severity Score; LAGISS: LAG plus ISS; LAGRTS: LAG plus RTS; LATRISS: LA plus TRISS.

${ }^{a}$ Binomial exact. 

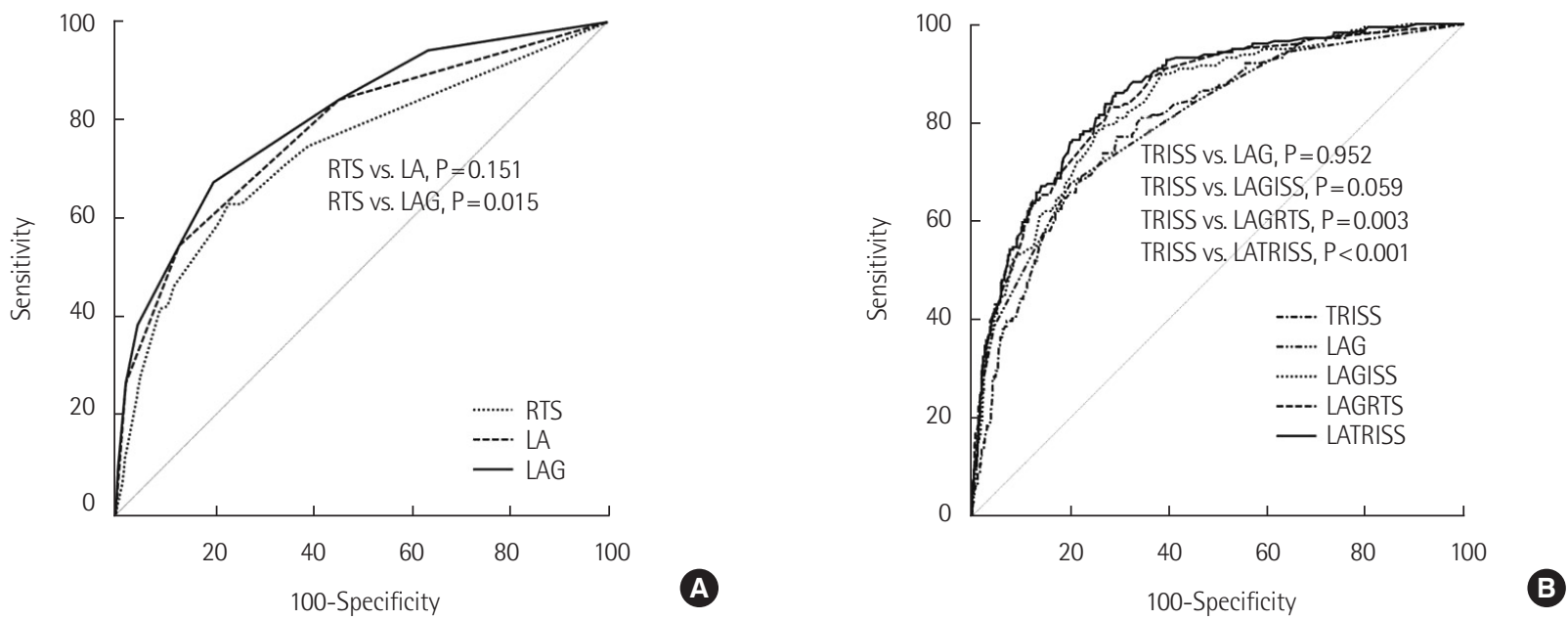

Figure 3. (A) Comparison of the areas under the receiver operator characteristic curves for mortality between the Revised Trauma Score (RTS) and other models using lactate (LA). (B) Comparison of the areas under the receiver operator characteristic curves for mortality between the Trauma and Injury Severity Score (TRISS) and other models using TRISS predictor variables plus lactate. LAG: lactate plus age; ISS: Injury Severity Score; LAGISS: LAG plus ISS; LAGRTS: LAG plus RTS; LATRISS: LA plus TRISS.

Table 3. Pairwise comparisons of ROC curves for the prediction models.

\begin{tabular}{|c|c|c|c|}
\hline Model & $\begin{array}{c}\text { Difference } \\
\text { between the } \\
\text { areas }\end{array}$ & $95 \% \mathrm{Cl}$ & P-value \\
\hline RTS vs. LA & 0.041 & -0.015 to 0.069 & 0.151 \\
\hline RTS vs. LAG & 0.067 & 0.013 to 0.121 & $0.015^{\mathrm{a}}$ \\
\hline TRISS vs. LAG & 0.001 & -0.044 to 0.047 & 0.952 \\
\hline TRISS vs. LAGISS & 0.035 & -0.001 to 0.071 & 0.059 \\
\hline TRISS vs. LAGRTS & 0.048 & 0.016 to 0.080 & $0.003^{\mathrm{a}}$ \\
\hline TRISS vs. LATRISS & 0.057 & 0.030 to 0.085 & $<0.001^{\mathrm{a}}$ \\
\hline LAGISS vs. LAGRTS & 0.013 & -0.005 to 0.031 & 0.152 \\
\hline LAGISS vs. LATRISS & 0.022 & 0.008 to 0.037 & $0.002^{\mathrm{a}}$ \\
\hline LAGRTS vs. LATRISS & 0.009 & 0.001 to 0.018 & $0.027^{\mathrm{a}}$ \\
\hline
\end{tabular}

ROC: receiver operating characteristic; Cl: confidence interval; RTS: Revised Trauma Score; LA: lactate; LAG: lactate plus age; TRISS: Trauma and Injury Severity Score; ISS: Injury Severity Score; LAGISS: LAG plus ISS; LAGRTS: LAG plus RTS; LATRISS: LA plus TRISS.

astatistically significant.

3, Figure 3A). However, this value was not significantly better than the value for the traditional TRISS $(0.801, \mathrm{P}=0.952)$ (Table 3, Figure 3B).

Regression analysis indicated that the model that incorporated age, ISS, and lactate level (LAGISS model) had a better predictive value than the TRISS model ( 0.836 vs. $0.801, \mathrm{P}=0.059$ ). In contrast, the LAGRTS model provided a significantly improved predictive value compared to the TRISS model ( 0.849 vs. $0.801, \mathrm{P}=0.003$ ). The LATRISS model provided the highest
Youden J value (0.568), with $86.0 \%$ sensitivity and $70.8 \%$ specificity at the cutoff point of 0.15 (Table 2). The LATRISS model also provided the highest predictive value, and this value was remarkably higher than that of the traditional TRISS method (difference, 0.057; $\mathrm{P}<0.001$ ) (Table 3, Figure 3B).

\section{DISCUSSION}

Since trauma management systems were established in the United States in the 1970s, several different systems have been suggested for scoring injury severity. However, only a few are widely used, such as the TRISS, which includes the ISS as an anatomical component, the RTS as a physiological component, and age as a comorbidity component. TRISS is useful for both blunt and penetrating trauma and can be used to predict the Ps in trauma cases $[10,15]$. However, its calculation requires accurate information regarding several variables (age, SBP, RR, GCS, and ISS), and missing or inaccurate data can lead to incorrect scores. Previous large-scale studies of the TRISS have revealed high proportions of missing data (11.3\%-23.3\%) [11, $13,14]$. In Korea, the immature trauma system is an additional problem, as there is no training or legal requirements for paramedics to assess initial physiological parameters, such as the GCS or SBP of injured patients, and most hospitals do not even perform ISS measurements. Thus, the accuracy of pre-hospital vital signs or GCS values is limited and/or these measurements are frequently omitted [16-18]. In addition, because pre-hospital triage for injured patients has not been well-established in Korea, $>50 \%$ of patients who should have been 
admitted directly to a trauma center were actually transferred to our facility from another hospital $[16,17]$. These issues make it extremely difficult to obtain accurate initial physiological data. In addition, ISS coding is only performed in 17 Korean trauma centers that were established after 2012, as time and resources are needed to train individuals to perform accurate coding [18]. Furthermore, the frequency of post-mortem examinations is much lower for Korean trauma cases than those in other countries [19,20], which further complicates AIS coding for patients who have died before or at their ER arrival. Therefore, TRISS measurements are difficult and have limited reliability in Korea, which is why we examined the utility of lactate levels for predicting trauma outcomes.

At our center, lactate level is assessed using arterial blood, and severe trauma cases (expected ISS score of $\geq 9$ ) undergo lactate testing upon ER arrival. The main drawbacks of this examination are its economic cost, invasive nature, and the time required for testing. However, it is a single test that can accurately reflect the patient's overall condition, regardless of the trauma's anatomical site or the assessment timepoint. In Korea, with its immature trauma system, even severe trauma patients are often transferred to another hospital without receiving appropriate definitive care at the first hospital. Thus, before being transferred, patients may receive massive intravenous fluid infusion, blood transfusion, or hemodynamic drugs, which can lead to discrepancies between their original vital signs and the vital signs that are measured at the trauma center or terminal hospital. These discrepancies can introduce error into the assigned TRISS value, which limits its predictive value. In contrast, almost all patients with severe trauma undergo blood sampling and lactate testing, which may be useful as a supplement or replacement for TRISS in predicting trauma outcomes. This possibility is supported by previous studies that have reported that lactate levels are associated with survival outcomes in trauma cases [21-26].

The present study examined data from a 5-year period at our center, and analyses of this data revealed a strong correlation between the initial lactate levels of patients with severe blunt trauma (ISS of $\geq 9$ ) and in-hospital mortality. Lactate testing was performed at a median interval of 12 minutes after ER arrival, and arterial blood was used for all cases. Patients who died had average lactate levels that were $3.1 \mathrm{mmol} / \mathrm{L}$ higher than those of patients who survived, and this difference was statistically significant. Thus, we coded patients into four groups according to lactate levels based on previous studies [25-28] and observed a positive correlation between initial lactate levels and mortality rate. Moreover, we performed logistic regres- sion to create lactate-based prediction models for trauma mortality. Logistic regression revealed that the lactate-based model provided a higher AUC value than the RTS-based model, although this difference was not significant. However, the model that used lactate levels and dichotomized age ( 55 years, the same age used for the TRISS calculation), provided a significantly higher AUC value than the RTS-based model. Therefore, initial lactate levels may be equally useful, or possibly superior, to traditional physiological parameters (e.g., SBP, RR, or GCS).

Previous reports have claimed that the standard for SBP should be adjusted to $110 \mathrm{~mm} \mathrm{Hg}$, rather than the $90 \mathrm{~mm} \mathrm{Hg}$ that is used in the RTS $[29,30]$. Similarly, the present study revealed that the median SBP was $110 \mathrm{~mm} \mathrm{Hg}$ in the non-survivor group, which may have contributed to the limited predictive value of the RTS. In addition, more precise measurement of the RR is needed, and its range should be modified, although our limited sample size prevented us from performing these additional analyses. Furthermore, we believe that classification of traditional vital signs (such as SBP) should eventually be reconsidered. Therefore, despite the invasive nature of lactate testing (relative to assessing blood pressure, pulse rate, or GCS), its utility in predicting trauma outcomes when incorporated in trauma scoring models potentially justifies its use.

Predictive values were similar when we compared the traditional TRISS model and a model that used lactate levels plus age. Thus, appropriate combination of lactate levels and traditional variables in a model will likely lead to better prediction of outcomes than the traditional TRISS model. We originally hypothesized that replacing the RTS with lactate levels in the TRISS model would improve the predictive value of the model; however, our analyses revealed that the improvement was only marginally significant $(\mathrm{P}=0.059)$. Nevertheless, combining the RTS and lactate levels without the ISS (an anatomical parameter) had superior predictive value to that of the TRISS model $(\mathrm{P}=0.003)$. Moreover, logistic regression using all of the variables (age, lactate, the ISS, and the RTS) provided the highest AUC value (0.859), which indicates that combining lactate levels with TRISS variables may be useful for developing a model with superior ability to predict trauma outcomes.

The present study had several limitations. First, patients were included only if they had experienced blunt trauma, undergone lactate testing at ER arrival, had complete physiological data, and had an ISS of $\geq 9$. Thus, our results are subject to selection bias, although this approach was necessary to compare the results to the traditional scoring system, as lactate levels are only tested at our center for patients with an ISS of 
$\geq 9$. Second, we did not consider whether patients had been transferred from another center, and all data regarding vital signs and GCS were obtained upon the patients' arrival at our ER. Thus, patients with no accurate GCS or RR (e.g., due to sedation or intubation before arrival) were excluded from the study. Third, we did not categorize cases based on the time of lactate testing or the reason for any delayed testing. Although most patients underwent testing relatively quickly (median interval of 12 minutes), approximately $5 \%$ of the patients underwent testing at $\geq 1$ hour after their arrival. Lastly, the present study is a single-center retrospective study in a country without a well-established trauma management system, and the findings may not be generalizable to other regions.

Our findings indicate that lactate testing upon ER arrival may help supplement traditional physiological parameters to predict trauma mortality in Korea. Furthermore, adding lactate levels improves the predictive ability of established trauma outcome prediction models, such as the RTS and TRISS. Nevertheless, these findings require validation using additional data from a large-scale, multi-center study.

\section{CONFLICT OF INTEREST}

No potential conflict of interest relevant to this article was reported.

\section{ACKNOWLEDGMENTS}

The authors thank our trauma registrar (Juryang Kim) and the hospital's Department of Electronic Medical Records for data collection and maintenance.

\section{ORCID}

Jonghwan Moon

https://orcid.org/0000-0002-4630-3301

Kyungjin Hwang https://orcid.org/0000-0002-5922-4186

Dukyong Yoon https://orcid.org/0000-0003-1635-8376

Kyoungwon Jung

\section{AUTHOR CONTRIBUTIONS}

Conceptualization: JM, KJ. Data curation \& Formal analysis: all authors. Methodology: all authors. Project administration \& Visualization: JM, KJ. Writing-original draft: JM, KJ, KH. Writing-review \& editing: all authors.

\section{REFERENCES}

1. Cook A, Weddle J, Baker S, Hosmer D, Glance L, Friedman L, et al. A comparison of the Injury Severity Score and the Trauma Mortality Prediction Model. J Trauma Acute Care Surg 2014;76:47-52.

2. Cassidy LD, Cook A, Ertl A, Gourlay D, Osler T. Is the Trauma Mortality Prediction Model (TMPM-ICD-9) a valid predictor of mortality in pediatric trauma patients? J Pediatr Surg 2014; 49:189-92.

3. Vivien B, Yeguiayan JM, Le Manach Y, Bonithon-Kopp C, Mirek S, Garrigue D, et al. The motor component does not convey all the mortality prediction capacity of the Glasgow Coma Scale in trauma patients. Am J Emerg Med 2012;30:1032-41.

4. Haider AH, Villegas CV, Saleem T, Efron DT, Stevens KA, Oyetunji TA, et al. Should the IDC-9 Trauma Mortality Prediction Model become the new paradigm for benchmarking trauma outcomes? J Trauma Acute Care Surg 2012;72:1695-701.

5. Moore L, Lavoie A, Turgeon AF, Abdous B, Le Sage N, Emond $\mathrm{M}$, et al. Improving trauma mortality prediction modeling for blunt trauma. J Trauma 2010;68:698-705.

6. Huber-Wagner S, Stegmaier J, Mathonia P, Paffrath T, Euler E, Mutschler W, et al. The sequential trauma score: a new instrument for the sequential mortality prediction in major trauma. Eur J Med Res 2010;15:185-95.

7. Glance LG, Osler TM, Mukamel DB, Meredith W, Wagner J, Dick AW. TMPM-ICD9: a trauma mortality prediction model based on ICD-9-CM codes. Ann Surg 2009;249:1032-9.

8. Osler T, Glance L, Buzas JS, Mukamel D, Wagner J, Dick A. A trauma mortality prediction model based on the anatomic injury scale. Ann Surg 2008;247:1041-8.

9. Marcin JP, Pollack MM. Triage scoring systems, severity of illness measures, and mortality prediction models in pediatric trauma. Crit Care Med 2002;30(11 Suppl):S457-67.

10. Boyd CR, Tolson MA, Copes WS. Evaluating trauma care: the TRISS method: trauma score and the injury severity score. J Trauma 1987;27:370-8.

11. Schluter PJ, Nathens A, Neal ML, Goble S, Cameron CM, Davey TM, et al. Trauma and Injury Severity Score (TRISS) coefficients 2009 revision. J Trauma 2010;68:761-70.

12. de Munter L, Polinder S, Lansink KW, Cnossen MC, Steyerberg EW, de Jongh MA. Mortality prediction models in the general trauma population: a systematic review. Injury 2017; 48:221-9

13. Schluter PJ. Trauma and Injury Severity Score (TRISS): is it time for variable re-categorisations and re-characterisations? Injury 2011;42:83-9. 
14. Champion HR, Copes WS, Sacco WJ, Lawnick MM, Keast SL, Bain LW Jr, et al. The Major Trauma Outcome Study: establishing national norms for trauma care. J Trauma 1990;30:135665.

15. Becher RD, Meredith JW, Kilgo PD. Injury severity scoring and outcomes research. In: Mattox KL, Moore EE, Feliciano DV, editors. Trauma. 7th ed. New York: McGraw-Hill; 2013. p. 77-90.

16. Jung K, Huh Y, Lee JC, Kim Y, Moon J, Youn SH, et al. The applicability of trauma and injury severity score for a blunt trauma population in Korea and a proposal of new models using score predictors. Yonsei Med J 2016;57:728-34.

17. Jung K, Lee JC, Park RW, Yoon D, Jung S, Kim Y, et al. The best prediction model for trauma outcomes of the current Korean population: a comparative study of three injury severity scoring systems. Korean J Crit Care Med 2016;31:221-8.

18. Jung K, Lee JC, Kim J. Injury severity scoring system for trauma patients and trauma outcomes research in Korea. J Acute Care Surg 2016;6:11-7.

19. Park JH, Na JY, Lee BW, Chung NE, Choi YS. A statistical analysis on forensic autopsies performed in Korea in 2015. Korean J Leg Med 2016;40:104-18.

20. Statistics Korea. Korean statistical information service [Internet]. Daejeon: Statistics Korea; 2018 [cited 2020 May 20]. Available from: http://kosis.kr/publication/publicationThema.do

21. Baxter J, Cranfield KR, Clark G, Harris T, Bloom B, Gray AJ. Do lactate levels in the emergency department predict outcome in adult trauma patients? A systematic review. J Trauma Acute Care Surg 2016;81:555-66.

22. Lewis CT, Naumann DN, Crombie N, Midwinter MJ. Prehospital point-of-care lactate following trauma: a systematic review. J Trauma Acute Care Surg 2016;81:748-55.
23. Parsikia A, Bones K, Kaplan M, Strain J, Leung PS, Ortiz J, et al. The predictive value of initial serum lactate in trauma patients. Shock 2014;42:199-204.

24. Ouellet JF, Roberts DJ, Tiruta C, Kirkpatrick AW, Mercado M, Trottier V, et al. Admission base deficit and lactate levels in Canadian patients with blunt trauma: are they useful markers of mortality? J Trauma Acute Care Surg 2012;72:1532-5.

25. Vandromme MJ, Griffin RL, Weinberg JA, Rue LW 3rd, Kerby JD. Lactate is a better predictor than systolic blood pressure for determining blood requirement and mortality: could prehospital measures improve trauma triage? J Am Coll Surg 2010; 210:861-9.

26. Callaway DW, Shapiro NI, Donnino MW, Baker C, Rosen CL. Serum lactate and base deficit as predictors of mortality in normotensive elderly blunt trauma patients. J Trauma 2009; 66:1040-4.

27. Mizushima Y, Ueno M, Watanabe H, Ishikawa K, Matsuoka T. Discrepancy between heart rate and makers of hypoperfusion is a predictor of mortality in trauma patients. J Trauma 2011;71:789-92.

28. Neville AL, Nemtsev D, Manasrah R, Bricker SD, Putnam BA. Mortality risk stratification in elderly trauma patients based on initial arterial lactate and base deficit levels. Am Surg 2011; 77:1337-41.

29. Hasler RM, Nuesch E, Jüni P, Bouamra O, Exadaktylos AK, Lecky F. Systolic blood pressure below $110 \mathrm{~mm} \mathrm{Hg}$ is associated with increased mortality in blunt major trauma patients: multicentre cohort study. Resuscitation 2011;82:1202-7.

30. Hasler RM, Nüesch E, Jüni P, Bouamra O, Exadaktylos AK, Lecky F. Systolic blood pressure below $110 \mathrm{mmHg}$ is associated with increased mortality in penetrating major trauma patients: Multicentre cohort study. Resuscitation 2012;83:476-81. 